7 Malave I, Layrisse Z, Layrisse M. Dose-dependent hyporreactivity to phytohemagglutinin in systemic lupus erythematosus. Cell Immunol 1975; 15: 231-236.

8 van der Velden AMT, Claessen AM, van Velzen-Blad H, et al. Development of $\mathrm{T}$ cell-mediated immunity after autologous stem cell transplantation: prolonged impairment of antigen-stimulated production of gamma-interferon. Bone Marrow Transplant 2007; 40: 261-266.
9 Vine MF, Stein L, Weigle K. Gender differences in response to the multitest CMI skin test in the general population. Ann Allergy Asthma Immunol 2000; 84: 445-450.

10 Strunk T, Hartel C, Temming P, et al. Erythropoietin inhibits cytokine production of neonatal and adult leukocytes. Acta Paediatr 2008; 97: 16-20.

\title{
Dermcidin identification from exhaled air for lung cancer diagnosis
}

\section{To the Editors:}

Exhaled breath condensate (EBC) analysis is a simple and truly non-intrusive approach to acquire information on understanding airway inflammation and other diseases of the respiratory system, such as tumourigenesis [1-3]. There are several striking advantages to utilising breath testing for screening purposes: 1) it does not influence airway function or cause inflammation [4]; 2) it can be performed repeatedly within short intervals [5];3) it is not significantly affected by age, sex or disease status [5]; and 4) it can be considered as a lung-specific analytic approach. Cancer cells have distinct properties from normal cells in that they may synthesise new proteins or change the protein expression levels during tumourigenesis [6]. A lot of soluble components of the lung exist in the epithelial lining fluid of alveoli [7]; therefore, the secreted new synthetic proteins can be digested into peptides under enzymatic processes. Subsequently, the small peptides have the possibility of adding to exhaled breath like other EBC compounds. The exploration of endogenous peptides, created by enzymatic cleavage of proteins in particular cellular environments, can result in relevant biomarker candidates [8, 9]. However, trace amounts of materials in EBC make detection a challenging task. This study was designed to determine the peptidome of EBC and to search for potential biomarkers for lung cancer diagnosis.

The study protocol was approved by the Institutional Review Board of the Kaohsiung Medical University Hospital (Kaohsiung, Taiwan). Patients with histological evidence of primary lung cancer who were admitted to the Division of Pulmonary and Critical Care Medicine (Dept of Internal Medicine, Kaohsiung Medical University Hospital), between January 2008 and August 2008 were enrolled in the study. We enrolled patients with squamous cell carcinoma, adenocarcinoma, small cell carcinoma, pneumonia and chronic obstructive pulmonary disease (COPD), as well as healthy subjects (table 1). The inclusion criteria included patients with newly diagnosed lung cancer before treatment. The diagnosis of lung cancer was confirmed by histological examinations of biopsy and/or cytology specimens obtained during fibreoptic bronchoscopy or with computed tomography-guided transthoracic needle aspiration biopsy. The stage of lung cancer was determined according to the staging system of the American Joint Committee on Cancer TNM classification. The early stage of lung cancer indicated stage I, II or IIIA. The late or advanced stage of lung cancer indicated stage IIIB and stage IV. Informed consent was obtained from each participant before enrolment in the study. In order to participate patients had to be aged $>18$ yrs. The control group of the study included subjects without lung cancer matched for socioeconomic group and age and who were nonsmokers, smokers and ex-smokers (defined as not having smoked for $\geqslant 1 \mathrm{yr}$ ). The controls were healthy or had had pneumonia or COPD during hospitalisation in the Kaohsiung Medical University Hospital (table 1). The exclusion criteria included: 1 ) patients refused to enter this study; 2) patients with lung cancer but experienced chemotherapy or radiotherapy; 3) patients with pulmonary tuberculosis; 4) patients with unclassified nonsmall cell lung cancer (NSCLC); 5) patients with other solid or haematological malignancy; and 6) patients with lung cancer but who could not open their mouth due to betel chewing or oral diseases.

The number of patients in each category and their ages and disease stages are listed in table 1. EBC collection was performed using an EcoScreen condenser (Jaeger, Wuzburg, Germany). Subjects were asked to breath at a normal frequency and tidal volume for $15 \mathrm{~min}$ while wearing a nose-clip. The exhaled air passed through a mouthpiece and a two-way nonrebreathing valve and was then frozen at $-20^{\circ} \mathrm{C}$. The condensates $(>1 \mathrm{~mL})$ were thawed and transferred to $1.5-\mathrm{mL}$ microtubes and immediately stored at $-70^{\circ} \mathrm{C}$. Bruker $\mathrm{Cu}$ magnetic beads (Bruker Daltonics, Leipzig, Germany) were used to purify EBC before mass spectrometer (MS) analysis. For MALDI-TOF (matrix-assisted laser depolarisation/ ionisation-time of flight) MS analysis, measurements were performed on a Bruker Ultraflex II MALDI-TOF/TOF mass spectrometer (Bruker Daltonics). For liquid chromatography (LC)/MS analysis, a nano-LC system coupled with Thermo LTQ-FTICR MS was used. Chromatographic separation was achieved by using self-packed $\mathrm{C}_{18}$ column at a split flow rate of $300 \mathrm{~mL} \cdot \mathrm{min}^{-1}$ in a 60 -min running cycle. For FTICR MS analysis, the full-scan mass survey was set at $\mathrm{m} / \mathrm{z} 320-1,800$ with mass resolution of 100,000 at $\mathrm{m} / \mathrm{z} 400$. Singly charged ions were rejected from MS/MS sequencing.

Based on MS/MS analysis and the MASCOT search, $\sim 20$ to 100 peptides that were deduced to be from $<10$ proteins were identified in each EBC sample. A total of 20 types of predicted 
TABLE 1 Characteristics of patients and the peptide E-R11 diagnosis

\begin{tabular}{|c|c|c|c|c|c|c|c|c|c|}
\hline & \multicolumn{2}{|c|}{ Sex $n$} & \multicolumn{2}{|c|}{ Age yrs } & \multicolumn{3}{|c|}{ E-R11 positive } & \multirow[t]{2}{*}{ Sensitivity \% } & \multirow[t]{2}{*}{ Specificity \% } \\
\hline & Male & Female & Male & Female & Non-cancer $^{+}$ & \multicolumn{2}{|c|}{ Cancer stage } & & \\
\hline Healthy & 8 & 4 & 40.4 & 55.5 & 0 (12) & & & & 100 \\
\hline Pneumonia & 8 & 6 & 51.6 & 48.0 & $2(14)$ & & & & 86 \\
\hline \multicolumn{10}{|l|}{ Carcinoma } \\
\hline Small cell & 5 & 0 & 58.6 & & & & $0(5)$ & 0 & \\
\hline Squamous cell & 10 & 0 & 70.2 & & & $2(3)$ & $3(7)$ & 50 & \\
\hline Adenocarcinoma & 16 & 16 & 59.6 & 61.5 & & $3(5)$ & $17(27)$ & 63 & \\
\hline
\end{tabular}

COPD: chronic obstructive pulmonary disease. ${ }^{\#}$ : using mass peak-area intensity $1.04 \times 10^{5}$ as a cut-off value for outcome measurement; ${ }^{\circ}$ : the early stage indicates stage I, II or IIIA and the late stage indicates stage IIIB or IV; ${ }^{+}$: data presented as $\mathrm{N}$ (case number)

proteins were found in all EBC samples. Among the identified peptides, the most abundant peptides were the fragments of keratin type I cytoskeletal 9 and type II cytoskeletal 1 proteins. The mass peak-area intensities of the relative peptides of both proteins showed no difference in the various groups of EBC. The most commonly identified peptides did not show a consistent expression pattern across the specific disease type or in every EBC sample. The dermcidin (DCD) peptide was the sole exception.

The $564.76 \mathrm{~m} / \mathrm{z}$ DCD peptide with a charge state of +2 had a conspicuous MS/MS spectrum for sequence determination, which was ENAGEDPGLAR aligning with amino acid residues 43 to 53 (fig. 1a). The reproducibility of MS analysis was estimated by three repeated measurements of various groups of EBC samples. The average constant of variance $(\mathrm{CV})$ values for health, squamous cell carcinoma and adenocarcinoma groups were $8.3 \%, 12.1 \%$ and $9 \%$, respectively, and the total average $\mathrm{CV}$ value was $9.6 \%$ for all measurements. The expression of $\mathrm{E}-$ R11 in every EBC sample was determined and the results showed that the NSCLC group, including the squamous cell carcinoma and adenocarcinoma group, had higher levels of ER11 than the healthy, small cell carcinoma and other respiratory disease groups (fig. 1b). The average mass peak-area intensities were $2.15 \times 10^{5}$ for squamous cell carcinoma and $2.46 \times 10^{5}$ for adenocarcinoma, but only $3.21 \times 10^{4}, 2.07 \times 10^{4}, 4.54 \times 10^{4}$ and $4.06 \times 10^{4}$ for healthy subjects, COPD, pneumonia and small cell carcinoma, respectively. The value of the area under the curve is 0.75 , which illustrates that E-R11 had a fair to good diagnostic power (fig. 1c). The diagnostic sensitivity and specificity show an optimum combination when the cut-off value of E-R11 mass peak-area intensity is approximately equal to $1 \times 10^{5}$. It is close to the value of the characteristic line with mass peak-area intensity of $1.04 \times 10^{5}$, which is the highest intensity of E-R11 in the healthy group (fig. 1b). With this cut-off threshold, all healthy subjects would not be diagnosed as NSCLC; however, a subset of COPD and pneumonia patients would have false diagnoses. The specificities for healthy subjects, COPD and pneumonia patients were $100 \%, 91 \%$ and $86 \%$, respectively, and for all non-cancer groups the specificity was $92 \%$. The sensitivities for squamous cell carcinoma and adenocarcinoma patients were $50 \%$ and $63 \%$, respectively, and $60 \%$ for all NSCLC groups. Using E-R11 for the diagnosis of NSCLC, the sensitivity and specificity showed no notable difference between the early and late cancer stages. Approximately $60 \%$ of early stage NSCLC patients (squamous cell carcinoma: two $(67 \%)$ out of three patients; adenocarcinoma: three $(60 \%)$ out of five patients) can be diagnosed with cancer (table 1). Our preliminary results indicate that E-R11 possesses a valuable potential to serve as a clinical biomarker for NSCLC early diagnosis.

To measure the E-R11 level in EBC we used a synthetic peptide as a standard for quantitative calibration. The linear relationship for peptide quantity and peak-area intensity was calculated as $y=1.04 x+2.64$ and an $R^{2}$ of 0.9999 , with the spots of peptide quantity $>100$ attomoles. According to this linear equation, mass peak-area intensity $1.04 \times 10^{5}$ was equal to 196 attomoles.

To validate the DCD expressed in lung cancer cells, the endogenous RNA expression of DCD in lung cancer patient tissues and lung cancer cell line samples was further determined. Two out of six squamous cell carcinoma and three out of six adenocarcinoma tissue samples had DCD RNA expression by RT-PCR analysis. Most of the paired normal tissue samples were found to have either no or very weak DCD expression. Endogenous RNA expression of DCD was also found in the lung cancer cell lines H520 (squamous cell carcinoma) and PC13 (adenocarcinoma). The lung fibroblast cell line, WI-38, and the bronchial epithelial cell line, BEAS-2B, were found to have no endogenous DCD expression. To characterise the biological activities of DCD in lung cancer cells we determined the functional consequences of DCD expression knockdown by using lentiviral vector delivered short hairpin (sh)RNA. Real-time quantitative PCR was used to assay the knockdown of DCD. The results indicate that both the H520 and PC13 cell lines could be infected at a high efficiency with DCD shRNA lentiviruses and that DCD shRNA could significantly knockdown the endogenous RNA expression of 

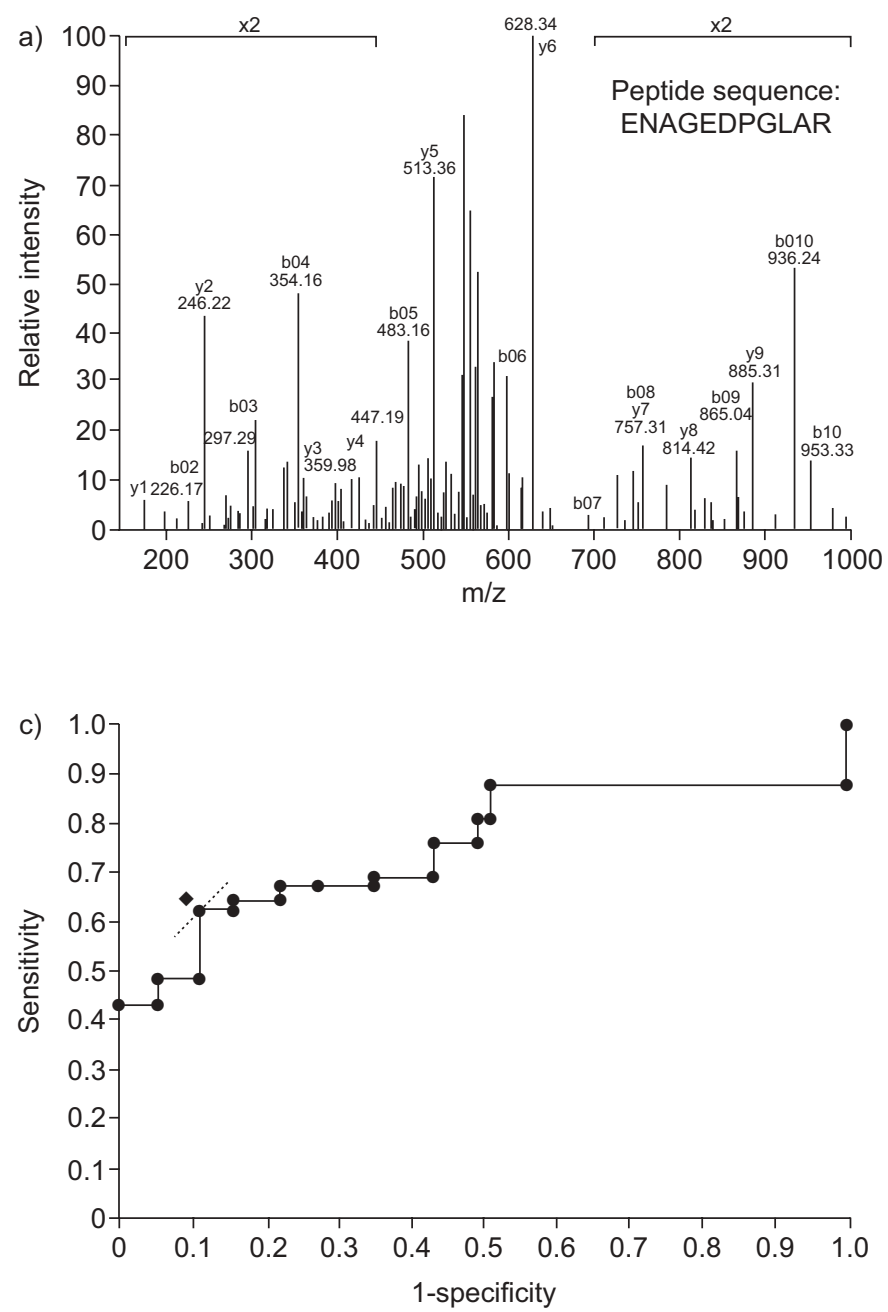

DCD in H520 $(\sim 60 \%)$ and PC13 $(\sim 70 \%)$. We further examined whether DCD expression knockdown resulted in growth reductions in normal or cancer cell lines. The results show $20-30 \%$ growth reductions in $\mathrm{H} 520$ and PC13 cells after DCD shRNA delivery, while no growth reduction was observed in normal fibroblast and epithelial cells.

In conclusion, EBC analysis combined with MS technique may become a valuable method for lung cancer screening and E-R11 may serve as a biomarker for NSCLC diagnosis.

\section{W.C. Chang*, , M.S. Huang", , C.J. Yang", , W.Y. Wang",}

T.C. Lai*, M. Hsiao* and C.H. Chen*

${ }^{*}$ Genomics Research Center, Academia Sinica, Taipei, and "Dept of Internal Medicine, Kaohsiung Medical University Hospital, Kaohsiung Medical University, Kaohsiung, Taiwan.

"These authors contributed equally.

Correspondence: C.H. Chen, Genomics Research Center, 128 Academia Road, Section 2, Nankang, Taipei 115, Taiwan. E-mail: winschen@gate.sinica.edu.tw

Support Statement: Funding was provided by the Genomics Research Center (Taipei, Taiwan), the National Health

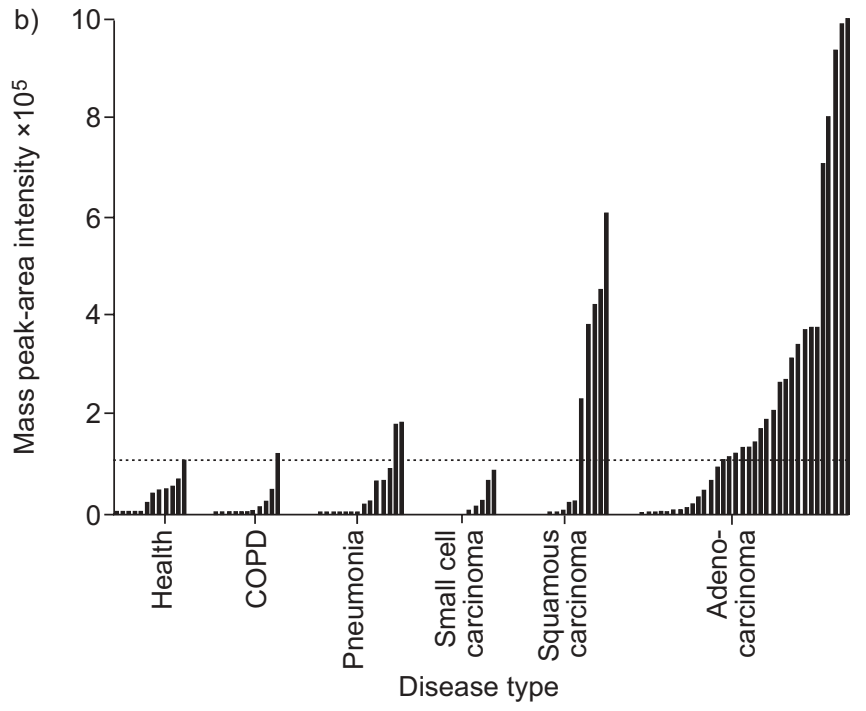

FIGURE 1. Characteristic analyses of E-R11. a) The E-R11 sequence and mass spectrometer (MS)/MS spectrum. The b-ion and y-ion fragments are shown. b) Expression of E-R11 in the exhaled breath condensate of healthy subjects and patients with different lung diseases. The characteristic line of mass peak-area intensity $1.04 \times 10^{5}$ represents the maximum value of E-R11 detected in the healthy subject group. COPD: chronic obstructive pulmonary disease. ……: 1.04. c) Receiver operating curve (ROC) analysis of E-R11 for nonsmall cell lung cancer diagnosis. Area under the curve: 0.75 . : the optimal Youden index showing the contact point of the ROC curve with the tangent of slope $1(\cdots \cdots \cdots)$.

Research Institute (Miaoli, Taiwan) and the National Science Council (grants NSC96-2314-B-037-062 and NSC98-2314-B-037053; Taipei, Taiwan).

Statement of Interest: None declared.

\section{REFERENCES}

1 Koutsokera A, Loukides S, Gourgoulianis KI, et al. Biomarkers in the exhaled breath condensate of healthy adults: mapping the path towards reference values. Curr Med Chem 2008; 15: 620-630.

2 Dalaveris E, Kerenidi T, Katsabeki-Katsafli A, et al. VEGF, TNFalpha and 8-isoprostane levels in exhaled breath condensate and serum of patients with lung cancer. Lung Cancer 2009; 64: 219-225.

3 Chan HP, Lewis C, Thomas PS. Exhaled breath analysis: novel approach for early detection of lung cancer. Lung Cancer 2009; 63: 164-168.

4 Carpagnano GE, Foschino Barbaro MP, Cagnazzo M, et al. Use of exhaled breath condensate in the study of airway inflammation after hypertonic saline solution challenge. Chest 2005; 128: 3159-3166.

5 Liu J, Thomas PS. Relationship between exhaled breath condensate volume and measurements of lung volumes. Respiration 2007; 74: 142-145.

6 Watkins SJ, Norbury CJ. Translation initiation and its deregulation during tumorigenesis. Br J Cancer 2002; 86: 1023-1027. 
7 Sabounchi-Schütt F, Aström J, Hellman U, et al. Changes in bronchoalveolar lavage fluid proteins in sarcoidosis: a proteomics approach. Eur Respir J 2003; 21: 414-420.

8 Chang WC, Hsu PI, Chen YY, et al. Observation of peptide differences between cancer and control in gastric juice. Proteomics Clin Appl 2008; 2: 55-62.
9 Petricoin EF, Belluco C, Araujo RP, et al. The blood peptidome: a higher dimension of information content for cancer biomarker discovery. Nat Rev Cancer 2006; 6: 961-967.

\section{An evaluation of a videobronchoscopy-based autofluorescence system in lung cancer}

\section{To the Editors:}

Autofluorescence (AF) has improved the sensitivity of bronchoscopy for pre-invasive cancer but has reduced specificity. Whether this applies to videobronchoscopy systems or depends on operator experience is unknown. We used a novel "slide-bank" model in order to evaluate the diagnostic performance of a video/AF bronchoscopy (AFI) system in lung cancer (refer to online supplementary material).

AF bronchoscopy systems are designed to enhance the detection of pre-invasive lesions. Abnormal bronchial epithelium fluoresces at an altered wavelength when illuminated by blue light. AF systems process and display these changes visually, revealing occult pre-invasive lesions.

Previously, the accuracy of AF systems in detecting preinvasive lesions and cancer has been compared with whitelight (WL) bronchoscopy [1, 2]. However, increases in sensitivity have been offset by reduced specificity, i.e. false positives. Furthermore, bronchoscopy and AF may be subject to significant, unreported, inter-operator variation. We evaluated an AFI system with experienced and inexperienced bronchoscopists to assess sensitivity/specificity, negative predictive value/positive predictive value (NPV/PPV) and interobserver agreement.

Patients undergoing investigation for haemoptysis/lung cancer underwent bronchoscopy. Those with an established diagnosis of lung cancer involving the central airways were excluded.

The study was approved by the local research ethics committee (Royal Brompton and Harefield Hospital, London, UK). All eligible patients who provided written informed consent underwent bronchoscopy performed by a single experienced operator (P.L. Shah) using the AFI system, under local anaesthetic with or without conscious sedation. The endobronchial tree was systematically examined under WL and then AF. The appearances were classified by the operator as normal, inflammatory, suspicious or definite malignancy. The still images of the endobronchial tree under both modes were captured and saved.

This article has supplementary material accessible from www.erj. ersjournals.com
We tested the AFI videobronchoscopy system (Olympus Medical Systems Corporation, Tokyo, Japan). This displays a high-quality WL image and can switch to AF mode by pressing a button. In AF mode the mucosa is illuminated by green and blue light. The combination of fluorescence and reflected green light is detected and processed into a composite image [3]. The on-screen image shows normal bronchial mucosa as green and neoplastic changes as magenta.

Histology from endobronchial biopsies were used as the gold standard for assessment. After completing an examination of the endobronchial tree, P.L. Shah took endobronchial biopsies from areas that appeared suspicious under either mode. Control areas that appeared normal under both modes were also biopsied in each patient. The pathologist (A.G. Nicholson) remained blinded to the bronchoscopic appearances and graded the biopsies according to the World Health Organization classification [4]. Intra-epithelial neoplasia was defined as moderate/severe dysplasia or carcinoma in situ [5]

Still images of biopsy sites were stored, creating a "slide-bank" library, and presented to individual observers as a slide show. They graded the appearance of the mucosa in each slide as either: normal, nonspecific abnormality/inflammatory, suspicious of cancer, or definite tumour. Five of the observers were experienced bronchoscopists who had performed >500 bronchoscopies and five were trainees with less experience $(<100$ bronchoscopies).

The histological findings were defined for statistical purposes as being positive (moderate dysplasia or worse) or negative. The real-time use of the AFI system by P.L. Shah was assessed by using the biopsy results to compare these to his grading of the bronchial mucosa under each AFI mode.

The slide show comprised both AF and WL images from each biopsied area, and paired testing was employed. The McNemar Chi-squared test for paired data (which confines analysis to divergent paired observations) was used to assess the sensitivity, specificity, PPV and NPV.

The difference between WL and AF modes and the effect of observer experience was quantified by the paired t-test for each value.

Inter-observer agreement was quantified using the weighted $\kappa$-coefficient of agreement (with quadratic weighting, such that $\kappa$-values are exactly synonymous with the intra-class correlation 